\title{
A Framework for Integrating the Distributed Hash Table (DHT) with an Enhanced Bloom's Filter in MANET
}

\author{
Ms. Renisha P Salim \\ Research Scholar \\ Bharathiyar University and Assistant Professor \\ Providence College of Engineering, India
}

\author{
Dr. Rajesh R \\ Associate Professor \\ Department of Computer Science \\ Christ (Deemed to be University), India
}

\begin{abstract}
MANET, a self-organizing, infrastructure-less, wireless network is a fast-growing technology in day-to-day life. There is a rapid growth in the area of mobile computing due to the extent of economical and huge availability of wireless devices which leads to the extensive analysis of the mobile ad-hoc network. It consists of the collection of wireless dynamic nodes. Due to this dynamic nature, the routing of packets in the MANET is a complex one. The integration of distributed hash table (DHT) in MANET is performed to enhance the overlay of routing. The node status updating in the centralized hash table creates the storage overhead. The bloom filter is a data structure that is a space-effective randomized one but it allows the falsepositive rates. However, this can be able to compensate for the issue of storage overhead in DHT (Distributed hash table). Hence, to overcome the storage overhead occurring in DHT, and reduce the false positives, the Bloom's filter is integrated with the DHT initially. Furthermore, the link stability is measured by the distance among mobile nodes. The optimal node selection should be done for the transmission of packets which is the lacking factor. If it fails to select the optimal path then the removal of malicious nodes may lead to the unwanted entry of nodes into the other clustering groups. Therefore, to solve this problem, the bloom's filter is modified for enhancing the link stability. The novelty of this proposed work is the integration of Bloom's filter with the Distributed Hash Table which provides good security on transmission data by removing false-positive errors and storage overhead.
\end{abstract}

Keywords-Mobile ad hoc network (MANET); distributed hash table (DHT); bloom's filter; link stability

\section{INTRODUCTION}

At present, Wireless communication shows a dynamic role in day-to-day life which leads to substantial growth in the mobile ad-hoc network (MANET). The MANETs are most commonly used in the area of military services, emergency services, sensing and gaming, education, the personal area network, and so on. It yields the benefits of centralized management of security, scalability, and good connectivity. It is the collection of various wireless dynamic nodes. Routing is the most significant method in the mobile ad-hoc network. The nodes that were present in the mobile ad-hoc network will perform the function of the router for transmitting the packets. Generally, the communication of the packet is done without any priory fixed network structure. Due to the dynamic nature of the mobile ad-hoc network, the routing of packets will be a complex one.

MANET is a kind of network with rapidly changing topology and are having a huge span with the capacity to connect hundreds [1] and thousands of nodes. The important feature of the mobile ad-hoc network is to identify the optimal path for the transmission of packets between the nodes. The nodes present in the mobile ad-hoc network are moving freely which affects its fixed infrastructure, hence it is necessary to introduce a dynamic network behavior.

The minimization of the network traffic is a significant part of constructing a MANET. For this, [2] a better QoS is essential to face this demand. Various mismatches [3] were identified in the construction of distributed hash tables like traffic overhead, high path stretching ratio, and long route. Also, in the traditional distributed hash table (DHT), each node of DHT will be proficient of maintain its table for the routing process. It also contains a list of communication links that are to be taken place without any awareness regarding the nodes of neighbor. The overlay of routing should be improved by integrating the distributed hash table with MANET.

The bloom filter is the randomized space-efficient structure of data for representing the [4] queries of support members. Conversely, the bloom filter is capable of allowing false positives. They are becoming very popular in the application of networks because of their concise size. The space-saving provided by this bloom filter will compensate for the issue of error occurrence probability.

Therefore, it is necessary to shrink the storage overhead and to diminish the false-positive rates of both the DHT and Bloom filters.

\section{A. Problem Identification}

The following problems are identified in the existing methodologies:

- The uncertainty of the route prediction is created due to the dynamic nature of the mobile nodes.

- The fault tolerance service is one of the major disputes in the MANET environment, provision of the optimal solution for this problem is necessary. 
- Generally, link stability is restrained by the distance between the mobile nodes. But it fails to choose the optimal node for the transmission of data without the consideration of speed, mobility, and the direction of mobility.

- Removal of malicious nodes will cause the nodes to enter into the other clustering groups.

In the proposed system, the hash table is combined with the bloom filter and the modification of the bloom filter is done for resolving the issues of storage overhead, false-positive errors and to improve the link stability.

\section{B. Objectives}

The main intention of this proposed system is as follows:

- To reduce the false-positive rates by combining the blooms filter with DHT.

- To enhance the link stability equation for computing the optimal routing path through modifying bloom's filter approach.

- The attacker and position possibility is included for improving the link stability.

- To maintain the centralized hash table with the bloom's filter.

The remaining portion of this paper is schematized as follows: Section II demonstrates the conventional works associated with the DHT, Bloom's filter for MANET in the wireless sensor networks. Section III illuminates the proposed (DHT-MBF) a novel distributed hash table integrated with the modified bloom filter. Section IV exhibits the performance study of the proposed mechanism and in conclusion, Section V concludes the proposed work.

\section{RELATED WORK}

This section deliberates the literature review of the routing protocols in MANET.

A Review was made on the routing protocols of the MANET in which the range of available routing protocols are discussed with their functionalities [5] which varies from protocols of early-stage to advanced protocols. It mainly focuses on developing and enhancing the MANET routing by Perkins. In general, ad-hoc networks offer a good potential in the circumstance at which access to the internet was not a chief requirement. Thus the evolution was made on the AODV protocol on analyzing the work depending upon the Multicast Ad-hoc on-demand Distance vector. However, this work needs some analysis on mobility-aware routing, Hierarchical routing, reliability-focused routing, and power-aware routing.

A survey was made on the distributed Hash tabledependent routing and the management [6] of data at the wireless sensor networks. The combination of the hash table over the wireless sensor network was made to manage the independent location of data and the identification of nodes. Various existing Hash table-based routing and the management of data protocols were described with their categories. Moreover, the detection of asymmetric link, bootstrapping and sensor dynamism was deliberated rarely which needs further analysis.

The analysis was made on the problems that were challenging in the mismatch among [7] resilience of overlay structure protocols and the physical networks. To overwhelm the difficulties of delay in average file discovery relay, the overhead of routing, high rate of average path-stretch, increased false-negative ratio, a distributed algorithm for exploiting the overlay and computing the subsequent logical identifier of the peer as defined in this work. However, there were some limitations like user anonymity, P2P partitioning of the network, load balancing, and free-riding issues.

The exploitation of 3-D structure was [8] made in MANET for the scalable routing is performed for avoiding the mismatch among the structure of logical identifier and the physical networks. This in turn preserves the issue of traffic overheads, the ratio of high path-stretch, and long routes. The approach of 3-D LIS was presented for managing the multi-paths at a destination node. The limitation of this work was Network merging and network partitioning.

A different methodology for the detection of service for MANETs was presented in which the protocols [9] of the service discovery efforts to overwhelm the incapability to abode the assets located at the network in which the node identity, its preceding knowledge, and also its ability is not accessible. The cross-layer approaches for the identification of service in MANET have been obtained to improve the procedure of discovery by the direct incorporation of the routing protocols. The novel service-oriented protocol for routing in MANETs was defined in this work. This method has improved success rates in relation to discovery and the throughput application in the densities of higher nodes.

An approach for maximizing the hash table throughput [10] at the network devices by combining the SRAM/DRAM Memory was performed. The Hash table is capable of forming a core component of a huge number of algorithms and network devices. It always requires a joint memory model as of its size at which some elements were kept in the slow memory and others were capable of storing in fast memory. The impacts of the memory speed difference with the choice of parameters were evaluated and the performances were traced. The employment of multiple-choice hashing was performed with the aid of combined memory. However, there were some limitations of this approach.

A QoS routing protocol depending on the link-state was presented which was established on the stability [11] of association for the mobile ad-hoc network. This approach was established to conserve the sustainable and stable path among the entire sets of nodes at a mobile ad-hoc network. The stability function was utilized as the main path for the selection criteria which was established on the degree of mobility in a node relation and its neighbor. For electing the constant and defensible MPR nodes and topologies this approach would be applied. The recompilation of MPR and routing tables were reduced by this mechanism. However, the metrics like packet loss and response time would be assured by applying this technique. Moreover, this approach needs some improvement 
by integrating with the other protocols of routing like DSR and AODV by executing in the real-time application.

An effectual and accessible dual region-based management of mobility for the mobile [12] ad-hoc network was presented for attaining the management of the location of nodes at the adhoc network. However, there were not at all selfish nodes or malicious nodes at the MANET for disrupting the management of mobility. Hence, it was necessary to investigate the management of trust protocols to choose the trustworthy nodes by enhancing the activity of dual-region management of location in the MANETs.

The routing technique was the most stimulating one in the mobile ad-hoc network [13] for its dynamic topology. There exist several types of routing protocols that are quite complex to identify the suitable routing protocols to the circumstances of the network. This paper provides the depiction of various routing protocols and the difference among them. The features of the MANET network were categorized as follows: the communication was through the wireless type, the functions of hosts and routers were done by the nodes, and also it was a bandwidth-constrained one, and so on. However, there were some limitations in different routing protocols which was complex to select various routing protocols along with the situations. Hence, it was necessary to face these contests for future widespread use.

An efficient addressing [14] protocol for the autoconfiguration of nodes at the ad-hoc networks was presented. The lightweight protocol for the configuration of the mobile ad-hoc network depends on the databases that were stored on the distributed address. From this approach, it was revealed that the address collisions were solved thereby reducing the control traffic on comparing traditional methodologies. The bloom filter was utilized in this approach. This approach was simple and diminishes the delay. However, there were some limitations in this kind of network.

A scheme of [15] congestion control in the case of a heterogeneous wireless ad-hoc network by using the self-adjust hybrid model was presented. The prediction of this heterogeneous wireless ad-hoc network was a complex one because of the occurrence of resource nodes in the distributed surroundings. The bloom filter was utilized for minimizing the error prediction which in turn reduces the fluctuations of load. The execution time was also increased. Anyhow this approach needs some improvement.

By using the [16] hierarchical bloom filter, the scalable content-based routing was presented for enabling the sharing, storing, and searching of information in the VANET. It offers a low rate of latency, ability to tackle mobility. This in turn offers a high completion rate as a result of the utilization of bloom's filter.

The addressing of [17] node replication was described and a different distributed hierarchical mechanism was introduced for the identification of node replication with the aid of the bloom filter approach. This provides an energy-efficient system in wireless networks. Moreover, this mechanism fails to validate the blooms filter through witnesses and in turn attain similar outcomes through identification.
The classification approach of the multi-label by using a large number of labels was [18] presented for the reduction of dimensional binary vectors. This scheme was robust to the inference complexity, sublinear training. The attitude was centered on the bloom filters mechanism. The computational difficulty was the shortcoming of this mechanism.

The optimization of traffic [19] in P2P centered on the congestion distance and DHT was stated which has been a major influence on the network of bearer type. The utilization of bandwidth networks has become a major problem for the user. The information collectors were assembled to the distributed hash table by the realization of information collectors and flow controllers. Therefore, by this scheme, the inter-autonomous system was reduced and the network traffic was diminished. To enhance the network traffic, this mechanism could be used by the other distributed system.

Abbas et al proposed a routing scheme [20] for limited configured devices. They uses on demand Ad-hoc distance vector algorithm for deriving the shortest path to the destination. Their model is validated in a simulated environment in Karachi and the experimental results claims that the performance is enhanced with minimal packet drops and constant throughput during the communication process. End-end communication delay is comparatively more which is a major drawback in their model.

Srilakshmi et al proposed a secure hybrid model for multipath routing [21] in the MANET. This work uses genetic algorithm with hill climbing which provides the optimal path. It uses prediction mechanism to choose the trust worth cluster heads and chooses alternate paths to reach the destination. It uses optimal energy for processing and is free from selective packet dropping attack.

\section{PROPOSED WORK}

This section deliberates the proposed method used for the enhancement of link stability for computing the optimal routing path on modifying Bloom's filter. The integration of Bloom's filter with the Distributed Hash Table is performed to reduce the storage overhead and occurrence of false positives.

Fig. 1 describes the overall flow of the prosed system in which the ID-based public key is generated and neighbor nodes are discovered. After sharing the public key, the hash table is generated. Then the bloom filter sends and receives the message after the discovery of nodes and updating of the distributed hash table. The received hash value must be verified in the DHT. If it does not match the condition then it is considered as the malicious node in the blocked list or else the data communication is performed for analyzing the link.

\section{A. Network Formation Module}

In the network module formation, the generation of IDbased public keys is carried out initially. The mobile nodes in the network will dynamically set a path temporarily to transmit the packet within them.

The discovery of neighborhood nodes is performed after the public key generation which in turn shares the public key to the neighborhood node. In MANET, the nodes are responsible for selecting the subset of the neighbor nodes to transmit the 
packet with minimum overhead. The development of a key management approach should be provided for the need to manage the routing table.

\section{B. Generation of Hash Table}

According to the ID-based public key that was created, the hash table $\mathrm{H}$ is generated. After that, the discovery of the route is carried out. The nodes that are located within one another's range will be communicated directly. The nodes that were located outside the ranges will be communicated through the other nodes for relaying the messages which should be performed with the routing protocols. Then the hash table is being updated for sending the message among nodes. The distributed hash table is updated according to the discovery of the route. The message that is to be transmitted should be coded or updated in the distributed hash table for security.

The routing of packets in MANET is a complex one because of its dynamic nature. Thus to improve the overlay of routing, the distributed hash table is integrated with the MANET. Each node in the traditional DHT maintains its routing table along with a list of links that are to be established for communication. Since they are unidirectional links it may unaware of neighbor nodes. From the neighbor list, the node chooses the routing path. The node status will be updated in the centralized portion of the hash table. This may lead to the storage overhead in the distributed hash table. It is necessary to reduce the storage overhead by integrating the bloom filter.

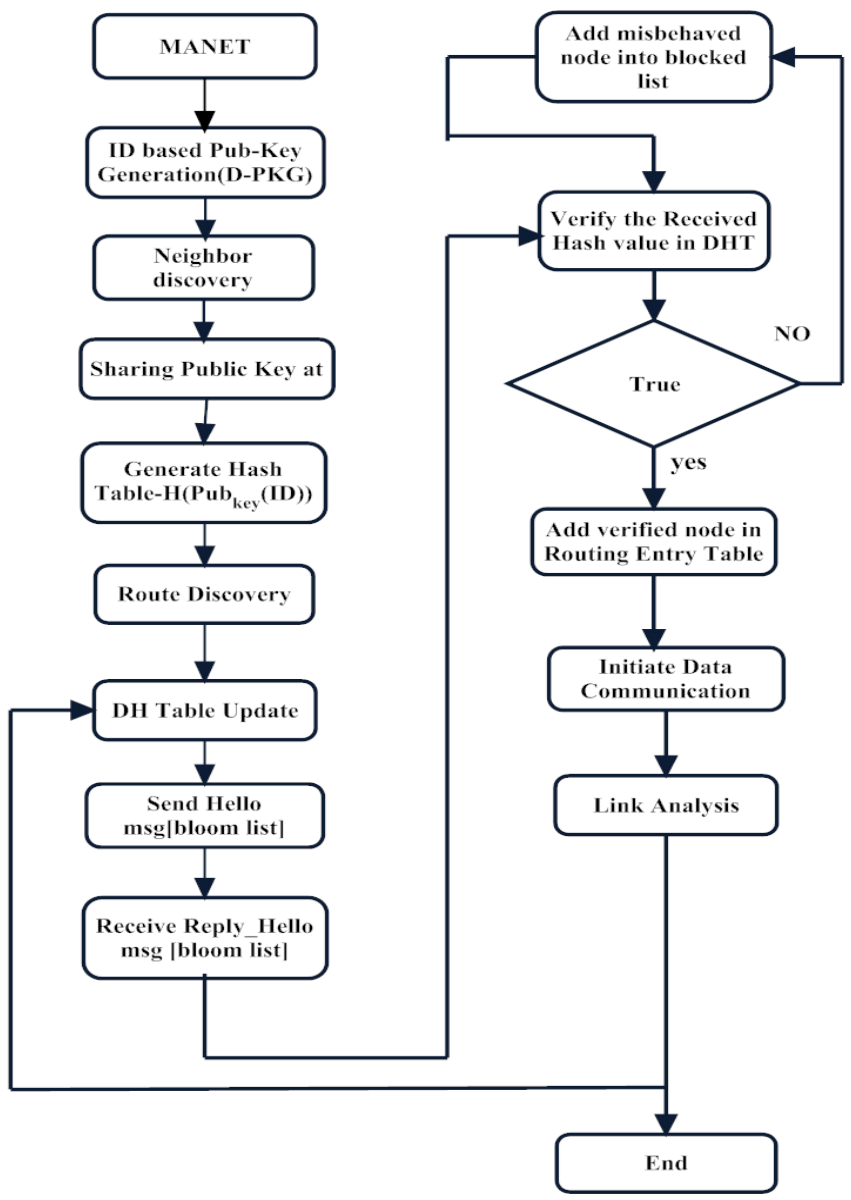

Fig. 1. Flow of the Proposed System.
The generation of the hash table was made by using node ID. The location and node ID are broadcasted and then the neighbor length is identified. The route is discovered by considering the source and destination id. The node should be sent by checking the path that is to be transmitted. This could be found using the own id of each node. For the next time of transmission, the packet should be transmitted through another hop. So the identification of hop is made to check if it is not the same hop through which the packet is already sent. Finally, the hash table is updated with the node status, the number of hops available for transmission, and the information regarding each hop.

Algorithm: Generation of Hash table using node ID

Step 1: Broadcasting the node id and location

Step 2: for $i$ : 1: length of neighbor

$$
\begin{aligned}
& p \leftarrow \operatorname{Hash}_{\text {key }}(\text { Id }) \otimes m \\
& \text { DHTable.id }\left[i, P u b_{\text {key }}\right] \leftarrow 1
\end{aligned}
$$

DHTable. $i d\left[i, H_{i}(p)\right] \leftarrow 1$

Step 3: if source $=$ Own (id) then

$t t l \leftarrow M A X T T L+1$

Next hop of $n$ (id)

list node $\rightarrow$ \{id $\}$ iii

Send_msg $(\{R w R E Q ;$ source ; dest ; list node; ttl $\}$,

UNICAST, next hop)

Set_timeout $(\{$ dest $\}$, WAITTIME)

end if

Step 4:

If Receive_msg(RwReQ,Source,dest, list_node,ttl $)$ then local_heat_level $\leftarrow \operatorname{Search}(G T A B$, dest $)$

if local_heat_level $==N O N E$ and $t t l>0$ then

next_hop $\leftarrow$ choose_random_neighbour ()

$t t l<t t l-1$

list_node $\leftarrow$ list_node $U$ myID

Send $_{m s g}\left(\left\{R w R E Q\right.\right.$, source $_{\text {dest }}$, list $\left.\left._{\text {node }}\right), t t l\right\}, U N I C A S T$, nexthop)

else if local_heat_level $\neq$ NONE then

Send_msg ( $\{$ FoHEAT, source, dest, list_node, local_heat_level $\}, B R O A D C A S T)$

endif

endif

Step 5:

if Receive msg(RoRep,soure,dest,prev $_{\text {node }}$, list $\left._{\text {node }}\right)$

then

next_hop $\leftarrow$ list_node

if source $==$ myID then

$A D D \_r o u t i n g \_e n t r y$ (prev_node,destination)

Forward_data_packet (next_hop)

else

next_hop $\leftarrow$ Last $\left(\right.$ list $\left._{\text {node }}\right)$

list_node $\leftarrow$ Remove_last(list_node)

Add_routing_entry(prev node, destination)

Send_msg( $\left\{\right.$ RoREP, source, dest, prev $v_{\text {node }}$, list $\left._{\text {node }}\right\}$,

endif

UNICAST, next_hop

endif 


\section{Bloom's Filter}

The data or message will be transmitted after the updating of the message in the distributed hash table. The message is transmitted with the help of the bloom list and is received with the same bloom filter. A bloom's filter is a randomized spaceefficient structure of data for representing the set and supporting membership queries. However, it may allow some false positives they may capable of offering some spaceefficient environment which compensates for the storage overhead occurring in DHT (Distributed hash table).

Bloom filters offer a tool for the identification of member set function which may include $\mathrm{N}$ number of array bits and various hash functions of $\mathrm{K}$. On installing the element, then the system is capable of sending an element to the various hash functions $\mathrm{K}$ and also sets the bits in an array by using the output values of the hash function. The system will check array bits that are corresponding to the hash function output values once the identification of the membership status of the element is set. After, the setting of all array bits to the respective hash function output values, then the system estimates the element as a member of all sets.

\section{Data Transmission}

After, the transmission of the message by bloom filter the verification is done in the received hash table to that of the DHT to check whether the sent and received messages are correctly matched. If this condition is satisfied then the verified node is added in the entry table of routing. If it fails to verify the condition, then it will be added in the blocked list as a misbehaved node which is then re-verified.

Finally, the data communication is initiated and the link stability is analyzed. Generally, link stability is measured by estimating the distance among the mobile nodes. The establishment of the connection is affected by the occurrence of a removed malicious node in another cluster. Link stability needs an optimal path for routing during the transmission of data. Depending on the real-time application, optimal path identification is an important process. To enhance the link stability, bloom's filter is modified. Hence, for the selection of optimal nodes, the reduction of false-positive rates and storage overhead is considered as a significant part.

Therefore, the integration of the bloom filter with the distributed hash table is much important to compute the optimal path, the reduction of storage overhead, and falsepositive rates. This is considered an effective solution.

\section{PERFormance ANALYSIS}

The performance analysis is described in this section by using the performance metrics like accuracy, delay, throughput, reliability, redundancy, average message overhead, and code rate.

\section{A. Simulation Analysis}

The simulation outcomes are analyzed by using the NS2 simulator. NS2 has turned into the most extensively used opensource network simulator, and one of the most commonly used network simulators. The simulation parameters are represented in the Table I provided here.
TABLE I. SIMULATION PARAMETERS

\begin{tabular}{|l|l|}
\hline Parameters & Values \\
\hline Number of nodes & 100 \\
\hline Topology size & 2500 X $1000 \mathrm{~m}^{2}$ \\
\hline Maximum Packet in Interface Queue & $100 \mathrm{bits}$ \\
\hline Data aggregation energy & $5 \mathrm{~nJ} / \mathrm{bit} /$ signal \\
\hline Protocol & AODV \\
\hline Duration of round & $20 \mathrm{~s}$ \\
\hline Initial energy & $100 \mathrm{~J}$ \\
\hline Idle Power & $0.1 \mathrm{~J}$ \\
\hline Received Power & $0.0645 \mathrm{~J}$ \\
\hline Transmit Power & $1.5 \mathrm{~J}$ \\
\hline Sleep Power & $0.01 \mathrm{~J}$ \\
\hline Duration Time & 99.001 ms \\
\hline Channel Type & Wireless Channel \\
\hline Radio Propagation Mode & Two Ray Ground \\
\hline Antenna Model & Omni Antenna \\
\hline Interface Queue Type & Queue/DropTail/PriQueue \\
\hline Network Interface Type & Mac/802_11 \\
\hline MAC Type & \\
\hline
\end{tabular}

\section{B. Performance Analysis}

This section provides the performance evidence of the proposed system about the performance metrics like packet delivery ratio, throughput, redundancy, code rate, total number of control packets, most reliable received bits, and the data packets that are sent.

1) Throughput: The number of data packets (measured in bits) that are directed over the total period of simulation is referred to as the throughput. It is also well-defined as the number of valuable bits to the per unit time progressed by the network to a definite destination from a certain source without considering the overhead and data packets that are transmitted. The throughput can be stated mathematically as follows:

Throughput $=\frac{\text { Number of data packets sent }(\text { bits })}{\text { simulation } \text { Time }(\operatorname{secs})}$

The simulation results are analyzed by simulation time vs throughput for both existing and proposed systems in Table II and Fig. 2 which specifies that the proposed system gives more throughput than the existing system.

TABLE II. Simulation Time Vs ThroughPUT

\begin{tabular}{|l|l|l|}
\hline Simulation time & $\begin{array}{l}\text { Throughput of existing } \\
\text { system }\end{array}$ & $\begin{array}{l}\text { Throughput of the } \\
\text { proposed system }\end{array}$ \\
\hline 5 & 350 & 379 \\
\hline 10 & 379 & 394 \\
\hline 15 & 395 & 413 \\
\hline 20 & 426 & 452 \\
\hline 25 & 489 & 502 \\
\hline
\end{tabular}




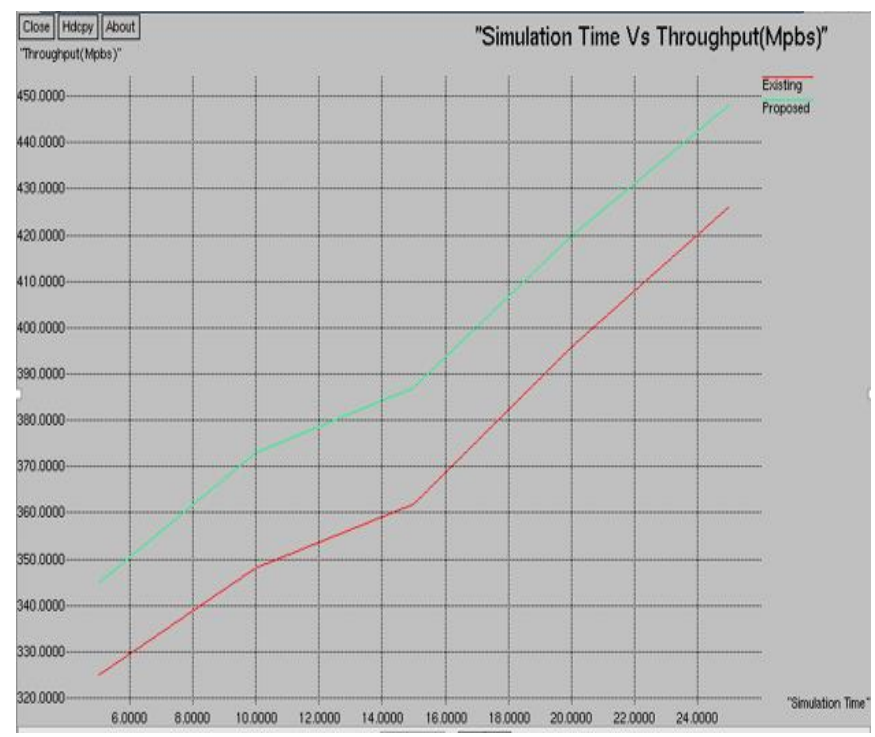

Fig. 2. Simulation Time vs Throughput.

2) Packet Delivery Ratio (PDR): The ratio of the sum of the packets that are received by the destination to the sum of packets that are generated is well-defined as the packet delivery ratio. In other disputes, it is the ratio of the number of data packets received by the receiver to the number of data packets transferred by the source. This can be stated as follows:

$P D R=\frac{\text { Sum of packets received by the destination }}{\text { Sum of packets generated in the source }}$

The simulation results are performed for simulation time vs packet delivery ratio in Table III and Fig. 3 which shows that the proposed system is offering high rate of packet delivery ratio than the existing system.

3) Average end-to-end delay: The average amount of time taken by the packet to reach the client node from the server node is known as the end-to-end delay of the packet. This can be stated as follows:

Delay $=\frac{\text { Number of packets sent }}{\text { simulation time }}$

The simulation outcome of the delay is provided for both existing and proposed systems in Table IV and Fig. 4. The delay of the proposed system increases regarding time.

TABLE III. SimUlation Time Vs Packet Delivery Ratio

\begin{tabular}{|l|l|l|}
\hline Simulation time & $\begin{array}{l}\text { Packet Delivery Ratio } \\
\text { of the existing system }\end{array}$ & $\begin{array}{l}\text { Packet Delivery Ratio } \\
\text { of the proposed system }\end{array}$ \\
\hline 5 & 17 & 20 \\
\hline 10 & 28 & 39 \\
\hline 15 & 45 & 57 \\
\hline 20 & 64 & 78 \\
\hline 25 & 88 & 95 \\
\hline
\end{tabular}

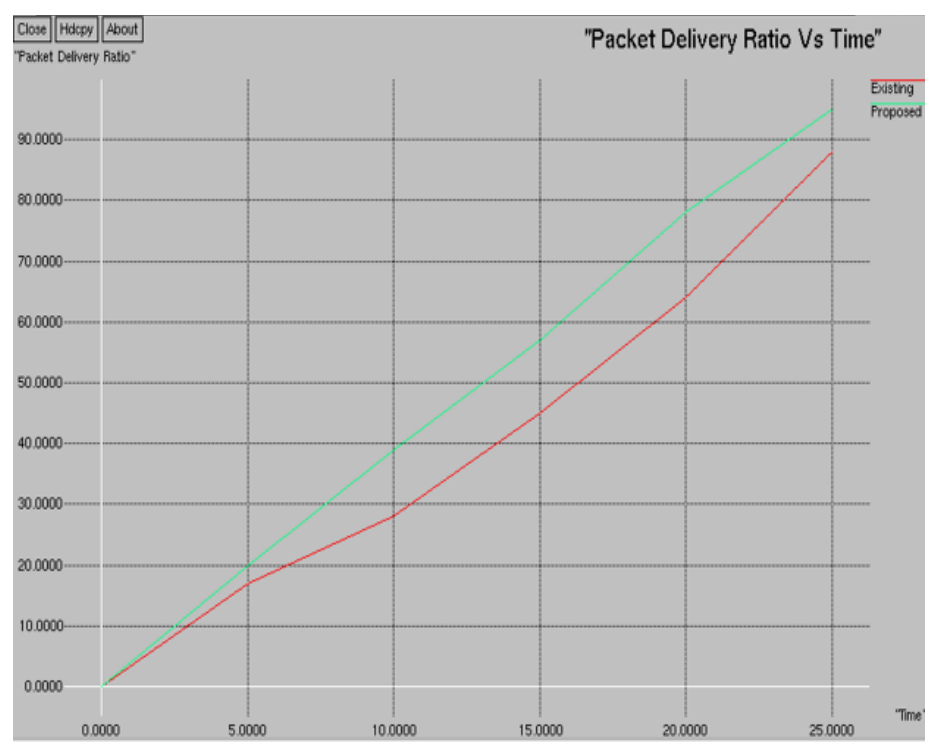

Fig. 3. Simulation Time vs Packet Delivery Ratio.

TABLE IV. Simulation Time vs Delay

\begin{tabular}{|l|l|l|}
\hline Simulation time & $\begin{array}{l}\text { Delay of existing } \\
\text { system }\end{array}$ & $\begin{array}{l}\text { Delay of the proposed } \\
\text { system }\end{array}$ \\
\hline 5 & 73 & 97 \\
\hline 10 & 174 & 194 \\
\hline 15 & 245 & 295 \\
\hline 20 & 366 & 399 \\
\hline 25 & 468 & 559 \\
\hline
\end{tabular}

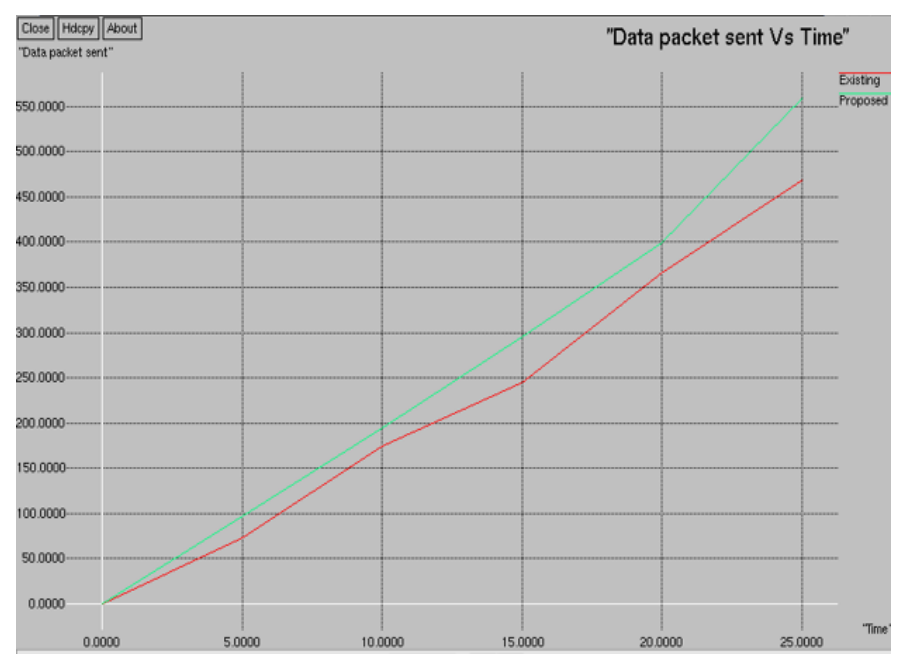

Fig. 4. Average End-to-End Delay vs Simulation Time.

4) Transmission time: The time is taken between the beginnings of transmission till the termination of message or packet transmission is termed as the transmission time. It is the ratio of packet size and the bit rate and can be expressed as follows:

Transmission time $=\frac{\text { packet size }}{\text { Bit rate }}$ 
The simulation outcome of the code rate is provided for both existing and proposed systems in Table V and Fig. 5. The transmission time of the proposed system increases regarding time.

5) Average message overhead: The ratio of the size of control packets to the total number of data packets that are transmitted successfully to the destination denotes the routing overhead.

By calculating the node and link failures primarily, the route discovery is attained rapidly. Henceforth the message overhead is condensed related to the existing mechanisms Table VI and Fig. 6.

\section{TABLE V. Simulation Time vs Code Rate}

\begin{tabular}{|l|l|l|}
\hline Simulation time & $\begin{array}{l}\text { Code rate of existing } \\
\text { system }\end{array}$ & $\begin{array}{l}\text { Code rate of the } \\
\text { proposed system }\end{array}$ \\
\hline 4 & 14 & 19 \\
\hline 8 & 26 & 38 \\
\hline 12 & 32 & 56 \\
\hline 16 & 39 & 73 \\
\hline 20 & 42 & 89 \\
\hline 24 & 48 & 95 \\
\hline
\end{tabular}

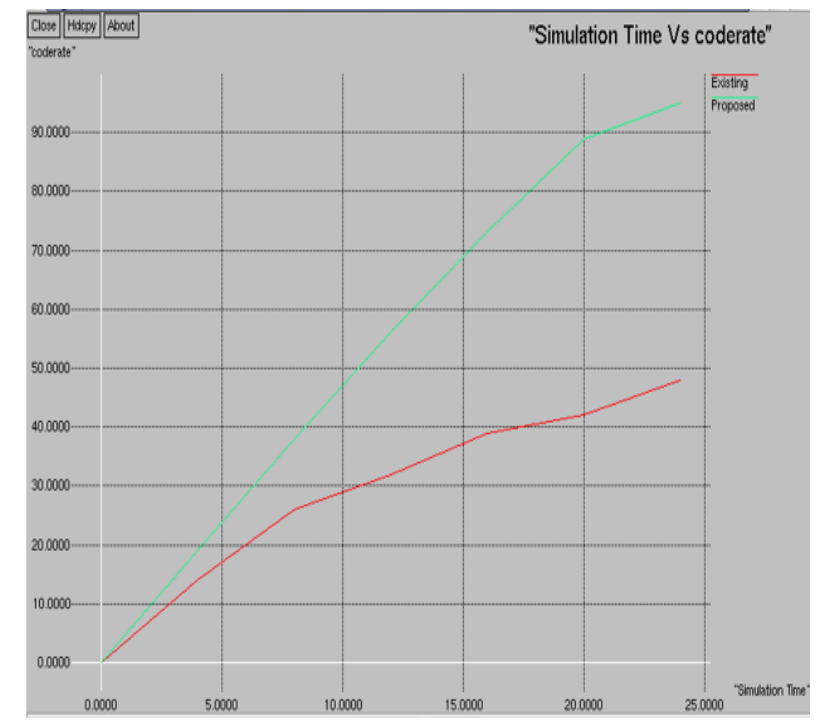

Fig. 5. Simulation Time vs Code Rate.

TABLE VI. Simulation Time Vs Total Number of CONTROL PACKETS

\begin{tabular}{|l|l|l|}
\hline Simulation time & $\begin{array}{l}\text { Total number of control } \\
\text { packets of existing } \\
\text { system }\end{array}$ & $\begin{array}{l}\text { Total number of control } \\
\text { packets of the proposed } \\
\text { system }\end{array}$ \\
\hline 4 & 8 & 8 \\
\hline 8 & 11 & 31 \\
\hline 12 & 19 & 39 \\
\hline 16 & 25 & 45 \\
\hline 20 & 29 & 49 \\
\hline 24 & 49 & 77 \\
\hline
\end{tabular}

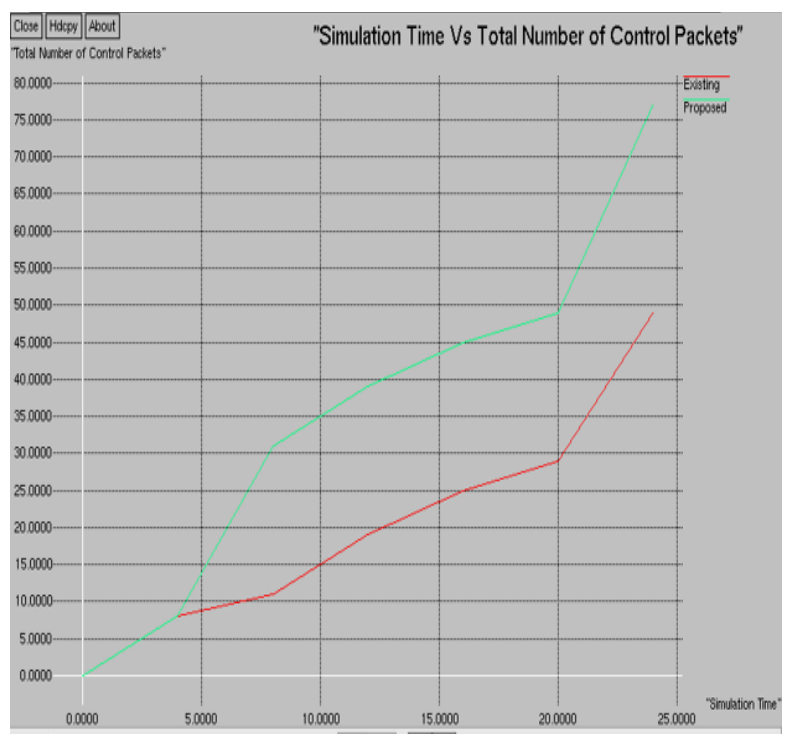

Fig. 6. Simulation Time vs Total Number of Control Packets.

6) Reliability: The ratio of most reliable received bits to the simulation time is identified as the reliability of the system. The simulation outcomes are performed by comparing the reliable bits of both proposed and existing mechanisms which yield a high rate of reliability for the proposed mechanism Table VII and Fig. 7.

TABLE VII. SimULATION TIME VS RELIABILITY

\begin{tabular}{|l|l|l|}
\hline Simulation time & $\begin{array}{l}\text { Reliability of existing } \\
\text { system }\end{array}$ & $\begin{array}{l}\text { Reliability of the } \\
\text { proposed system }\end{array}$ \\
\hline 0 & 0 & 0 \\
\hline 5 & 65 & 79 \\
\hline 10 & 159 & 185 \\
\hline 15 & 229 & 279 \\
\hline 20 & 348 & 369 \\
\hline 25 & 427 & 542 \\
\hline
\end{tabular}

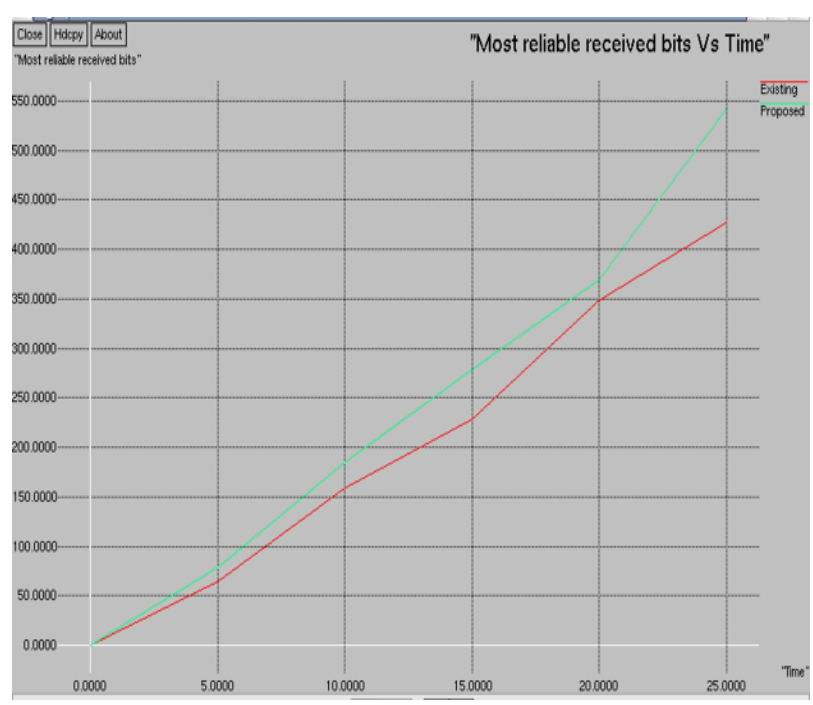

Fig. 7. Simulation Time vs Reliability. 
7) Redundancy: Redundancy is well-defined as the rate of occurrence of a fault in the network system which is restrained concerning the time of the simulation.

By this simulation analysis Table VIII and Fig. 8 shows that the existence of faulty nodes is less in the proposed system than the existing mechanism.

TABLE VIII. SimULATION Time Vs REDUNDANCY

\begin{tabular}{|l|l|l|}
\hline Simulation time & $\begin{array}{l}\text { Redundancy of } \\
\text { existing system }\end{array}$ & $\begin{array}{l}\text { Redundancy of the proposed } \\
\text { system }\end{array}$ \\
\hline 0 & 0 & 0 \\
\hline 3 & 0.255 & 0.0023 \\
\hline 6 & 0.588 & 0.0067 \\
\hline 9 & 1.99 & 0.0133 \\
\hline 15 & 3.65 & 0.033 \\
\hline 20 & 6.77 & 0.048 \\
\hline 23 & 9.44 & 0.069 \\
\hline 25 & 12.54 & 0.115 \\
\hline
\end{tabular}

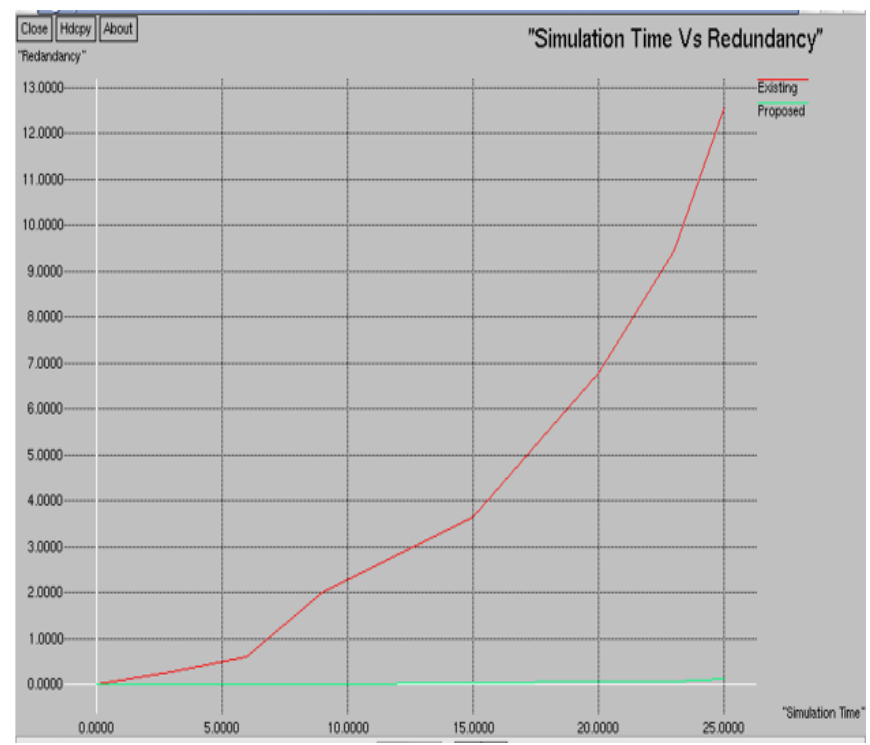

Fig. 8. Simulation Time vs Redundancy.

\section{CONCLUSION}

MANET was an infrastructure-less fast-growing wireless sensor network in which the routing protocol was complex due to its dynamic nature. The distributed hash table was used to secure the communication of data in which the updating of node status was performed. This in turn causes a storage overhead. The bloom's filter was utilized to compensate the storage overhead of the DHT which was capable of allowing false-positive rates. Hence, the integration of Distributed hash table was done with the bloom filter (DHT-MBF) to compensate for the storage overhead and reduction of falsepositive rates. The link stability was considered as an important factor in which the removal of malicious nodes would cause unwanted entry into the other clustering nodes. Therefore, the link stability was enhanced by the modification of Bloom's filter.
The performance analysis was made and the results were obtained by the simulation which shows that our proposed (EDHT-MBF) Enhanced distributed hash table with the integration of modified bloom's filter reduces the storage overhead and occurrence of false positives thereby increasing the security of data communication.

\section{REFERENCES}

[1] N. Jain and Y. Chaba, "Simulation based performance analysis of zone routing protocol in Manet," International Journal of Computer Applications, vol. 88, 2014.

[2] C. Cameron, I. Khalil, and Z. Tari, "An ID-based approach to the caching and distribution of peer-to-peer, proxy-based video content," Journal of Network and Computer Applications, vol. 37, pp. 293-314, 2014.

[3] Y. Zhang, D. Li, Z. Sun, F. Zhao, J. Su, and X. Lu, "CSR: Classified Source Routing in DHT-Based Networks," IEEE Transactions on Cloud Computing, 2015.

[4] L. Carrea, A. Vernitski, and M. Reed, "Optimized hash for network path encoding with minimized false positives," Computer networks, vol. 58, pp. 180-191, 2014.

[5] A. Hinds, M. Ngulube, S. Zhu, and H. Al-Aqrabi, "A review of routing protocols for mobile ad-hoc networks (manet)," International journal of information and education technology, vol. 3, p. 1, 2013.

[6] G. Fersi, W. Louati, and M. B. Jemaa, "Distributed Hash table-based routing and data management in wireless sensor networks: a survey," Wireless networks, vol. 19, pp. 219-236, 2013.

[7] S. A. Abid, M. Othman, and N. Shah, "3D P2P overlay over MANETs," Computer Networks, vol. 64, pp. 89-111, 2014.

[8] S. A. Abid, M. Othman, and N. Shah, "Exploiting 3D structure for scalable routing in MANETs," IEEE Communications Letters, vol. 17, pp. 2056-2059, 2013.

[9] W. Kenny and S. Weber, "Below cross-layer: an alternative approach to service discovery for MANETs," in International Conference on Ad Hoc Networks, 2012, pp. 212-225.

[10] Y. Kanizo, D. Hay, and I. Keslassy, "Maximizing the throughput of hash tables in network devices with combined SRAM/DRAM memory," IEEE Transactions on Parallel and Distributed Systems, vol. 26, pp. 796$809,2015$.

[11] A. Moussaoui, F. Semchedine, and A. Boukerram, "A link-state QoS routing protocol based on link stability for Mobile Ad hoc Networks," Journal of Network and Computer Applications, vol. 39, pp. 117-125, 2014.

[12] R. Chen, Y. Li, R. Mitchell, and D.-C. Wang, "Scalable and efficient dual-region based mobility management for ad hoc networks," Ad Hoc Networks, vol. 23, pp. 52-64, 2014.

[13] D. S. Dhenakaran and A. Parvathavarthini, "An overview of routing protocols in mobile ad-hoc network," International Journal of Advanced Research in Computer Science and Software Engineering, vol. 3, 2013.

[14] N. C. Fernandes, M. D. D. Moreira, and O. C. M. B. Duarte, "An efficient and robust addressing protocol for node autoconfiguration in ad hoc networks," IEEE/ACM Transactions on Networking (TON), vol. 21, pp. 845-856, 2013.

[15] M. Rajesh and J. Gnanasekar, "Congestion Control Scheme for Heterogeneous Wireless Ad Hoc Networks Using Self-Adjust Hybrid Model," International Journal of Pure and Applied Mathematics, vol. 116, pp. 537-547, 2017.

[16] Y. T. Yu, M. Gerla, and M. Sanadidi, "Scalable VANET content routing using hierarchical bloom filters," Wireless Communications and Mobile Computing, vol. 15, pp. 1001-1014, 2015.

[17] W. Znaidi, M. Minier, and S. Ubéda, "Hierarchical node replication attacks detection in wireless sensor networks," International Journal of Distributed Sensor Networks, vol. 9, p. 745069, 2013.

[18] M. M. Cisse, N. Usunier, T. Artieres, and P. Gallinari, "Robust bloom filters for large multilabel classification tasks," in Advances in Neural Information Processing Systems, 2013, pp. 1851-1859. 
[19] Q. He, Q. Dong, B. Zhao, Y. Wang, and B. Qiang, "P2P Traffic Optimization based on Congestion Distance and DHT," J. Internet Serv. Inf. Secur., vol. 6, pp. 53-69, 2016.

[20] T. Abbas, F. Qamar, M. N. Hindia, R. Hassan, I. Ahmed and M. I. Aslam, "Performance Analysis of Ad Hoc on-Demand Distance Vector Routing Protocol for MANET," 2020 IEEE Student Conference on Research and Development (SCOReD), 2020, pp. 194-199.
[21] U. Srilakshmi, N. Veeraiah, Y. Alotaibi, S. A. Alghamdi, O. I. Khalaf and B. V. Subbayamma, "An Improved Hybrid Secure Multipath Routing Protocol for MANET," in IEEE Access, vol. 9, pp. 163043163053, 2021. 